\title{
Complejidad estructural de los manglares de Playa Blanca, Escondido y Rincón de Osa, Golfo Dulce, Costa Rica
}

\author{
Jimena Samper-Villarreal ${ }^{2}$ \& Ana Margarita Silva-Benavides ${ }^{1,2 *}$ \\ 1. Escuela de Biología, Universidad de Costa Rica, San Pedro, San José 2060, Costa Rica; ana.silva@ucr.ac.cr \\ 2. Centro de Investigación en Ciencias del Mar y Limnología (CIMAR), Ciudad de la Investigación, Universidad de \\ Costa Rica, San Pedro, San José 2060, Costa Rica; jimena.samper@gmail.com \\ * Correspondencia
}

Recibido 11-VIII-2014. Corregido 20-XI-2014. Aceptado 22-XII-2014.

\begin{abstract}
Structural complexity of mangroves in Playa Blanca, Escondido and Rincón de Osa, Golfo Dulce, Costa Rica. This study reports the structural characteristics and distribution of species in the mangrove forests of Playa Blanca, Escondido and Rincón de Osa in Golfo Dulce, South Pacific of Costa Rica. Multiple lineal transects were carried out at each of the mangrove forests. Every 10 meters along each transect the diameter at breast height, basal area, and height was determined for all mangrove trees within a $5 \times 5$ meter square plot. Sediment and interstitial water (salinity) samples were also collected at each plot. Total density of the stand was determined, as were the density of each mangrove species, the importance value and basal area per species, and general complexity indices for each mangrove forest. The dominant species at all three mangroves were Rhizophora racemosa and Pelliciera rhizophorae. Forest structure was more similar between Playa Blanca and Rincón mangroves than with Escondido. Playa Blanca was the forest with the tallest canopy height and lowest density, while Escondido had the highest density and the shortest canopy height. Escondido was also the forest with the least number of species and the only one where Conocarpus erectus was present. Species composition and dominance at these mangroves coincides with that reported for the region. However, the variation in structure and complexity among the three mangroves is greater than the commonalities with mangroves in the region. Topographical and historical analysis of these mangroves could further elucidate the present structure and complexity variation among them. Rev. Biol. Trop. 63 (Suppl. 1): 199-208. Epub 2015 April 01.
\end{abstract}

Key words: Mangrove structure, Rhizophora, Pelliciera, Avicennia, Laguncularia, Conocarpus, Osa Peninsula, Golfo Dulce, Costa Rica.

Los bosques de manglar son ecosistemas costeros de gran importancia, que cumplen funciones críticas como áreas de crianza para múltiples especies marinas, muchas de interés comercial, sitios de consolidación y filtración de sedimento y nutrimentos, protección de la zona costera evitando su erosión y su función como sumideros de carbono (Hogarth, 1999; Kathiresan \& Qasim, 2005; Donato et al., 2011). Estos ecosistemas costeros se encuentran en zonas entre mareas de las regiones tropicales y subtropicales (Tomlinson, 1994; Spalding, Spalding, Kainuma, \& Collins, 2010). Taxonómicamente, los manglares consisten en un grupo diverso de comunidades leñosas pertenecientes a varias familias de angiospermas, no relacionadas entre sí, con adaptaciones especiales a condiciones salinas (Hogarth, 1999).

La complejidad y la estructura de los bosques de mangle es el resultado de las condiciones climáticas, hidrológicas y físicas predominantes en estos ecosistemas, en conjunto con las características biológicas del bosque (Jiménez \& Soto, 1985). La precipitación y la temperatura son factores climáticos críticos en determinar la composición y la distribución geográfica general de las especies de mangle. 
Sin embargo, la distribución local de estos bosques está influenciada en gran parte por factores como tipo de suelo, pendiente, inundación mareal y escorrentía (Satyanarayana, Raman, Dehairs, Kalavati, \& Chandramohan, 2002). De igual manera, estas fuerzas ambientales que actúan sobre los manglares varían en periodicidad e intensidad (Cintrón-Moreno \& SchaefferNovelli, 1992) y la respuesta por parte de la vegetación de manglar a la variabilidad en dichas presiones ambientales está limitada por la flexibilidad genética de las especies (Cintrón \& Schaeffer-Novelli, 1983; Cintrón-Moreno \& Schaeffer-Novelli, 1992).

En Costa Rica, el 99\% de los manglares se ubican en la costa Pacífica, con una cobertura total estimada de 41000 ha de costa (Pizarro \& Angulo, 1994; Zamora-Trejos \& Cortés, 2009). El MINAET (2013), informa que para este año, el porcentaje de cobertura de los bosques de mangle en Costa Rica comprende un $0.7 \%$ de todos los tipos de bosque que existen en el país. Se han realizado varios estudios en diversos manglares de esta costa, especialmente en el Pacífico Norte (Zamora-Trejos \& Cortés, 2009), enfocados en la descripción de las especies (Córdoba-Muñoz, RomeroAraya, \& Windevoxhel-Lora, 1998) y estudio de la complejidad estructural de la vegetación (Pool, Snedaker, \& Lugo, 1977; Soto \& Jiménez, 1982; Jiménez \& Soto, 1985; SamperVillarreal, Cortés, \& Benavides-Varela, 2012). Descripciones realizadas en los manglares a lo largo de la costa Pacífica de Costa Rica, indican diferencias en la estructura del bosque y composición de especies, señalando tres regiones específicas: Norte, Central y Sur, cada una caracterizándose por poseer diferentes condiciones ambientales que influyen directamente en las especies dominantes y su estructura (Jiménez \& Soto 1985, Pizarro, Piedra, Bravo, Asch, \& Asch 2004, Zamora-Trejos $\&$ Cortés 2009).

Los bosques de mangle que se encuentran en la región interna de Golfo Dulce (Pacífico Sur), en comparación con el Pacífico Norte, han recibido poca atención especialmente relacionado con el tema de la estructura del bosque
(Mainardi, 1996; Jiménez, 1999; Silva, 2005; Nielsen-Muñoz \& Quesada-Alpízar, 2006; Quesada-Alpízar \& Cortés, 2006; Silva, 2009). El presente estudio analiza las características estructurales, distribución de las especies de mangle y complejidad de los bosques de Playa Blanca, Escondido y Rincón de Osa en Golfo Dulce, Costa Rica.

\section{MATERIALES Y MÉTODOS}

Sitio de Estudio: Los bosques de mangle de Playa Blanca, Escondido y Rincón de Osa se encuentran en la zona del Pacífico Sur de Costa Rica; en la parte interna de Golfo Dulce. El manglar de Playa Blanca ( $8^{\circ} 39^{\prime} 4^{\prime \prime}$ $\mathrm{N}-83^{\circ} 26^{\prime} 3^{\prime \prime} \mathrm{W}$ ), se encuentra localizado al norte de Puerto Jiménez, entre los ríos Conte y La Chanchera, con una extensión de cuenca estimada en 2536.6ha (Echeverría-Sáenz, 2009). Los manglares de Escondido ( $8^{\circ} 41^{\prime} 34^{\prime \prime}$ $\left.\mathrm{N}-83^{\circ} 28^{\prime} 42^{\prime \prime} \mathrm{W}\right)$ y Rincón (843'6” N $\left.83^{\circ} 28^{\prime} 53^{\prime \prime} \mathrm{W}\right)$ reciben el caudal de la cuenca del río Rincón y sus afluentes (21418.6ha) (Echeverría-Sáenz, 2009).

La época seca comprende de diciembre a abril, con una época lluviosa pronunciada el resto del año. La precipitación total promedio en esta región es una de de las más altas en el país, entre 3500 y $5500 \mathrm{~mm}_{\text {año }}^{-1}$ (Instituto Meteorológico Nacional de Costa Rica (IMN), 2013). El Golfo Dulce en general, presenta poca alteración antropogénica; sin embargo, los cultivos de arroz en zonas aledañas y la extracción de piangüas por parte de pobladores locales, constituyen las principales actividades que pueden impactar negativamente los manglares del Golfo.

\section{Diseño de muestreo}

Distribución de las especies de mangle y estructura forestal: En cada manglar se realizaron múltiples transectos lineales perpendiculares a la costa, con un mínimo de $100 \mathrm{~m}$ de longitud cada uno, durante los años 2008 y 2009. Sobre cada transecto analizado se establecieron parcelas cuadradas de $5 \times 5 \mathrm{~m}$, 
dispuestas cada $10 \mathrm{~m}$ a lo largo del transecto hasta el interior del manglar estudiado. El número de parcelas analizadas en cada manglar dependió de la longitud y número total de transectos realizados. La distancia entre transectos varió según las posibilidades de acceso. En el manglar de Playa Blanca se analizaron 15 transectos (185 parcelas), siete transectos en el manglar de Rincón (77 parcelas) y seis transectos en el manglar de Escondido (87 parcelas).

En cada parcela se identificó las especies de mangle presentes y se estimó la altura de cada árbol (m) y el diámetro a la altura del pecho (DAP) de cada individuo a $1.20 \mathrm{~m}$ del suelo o arriba de la raíz más alta en el caso de las raíces aéreas de Rhizophora racemosa y Rhizophora mangle, de acuerdo a Cintrón y Shaeffer-Novelli (1984) y CARICOMP (2001). Los árboles localizados en los bordes de las parcelas se consideraron en el muestreo, condicionados que la mitad del tallo se encontrara dentro del cuadrado. Se anotaron las Familias de la vegetación asociada observada durante los muestreos.

Se cuantificó el área basal para cada especie de mangle, la densidad de cada especie y la densidad total de árboles de mangle en cada sitio. Se analizó la complejidad estructural de cada manglar determinando el valor de importancia para cada especie (densidad relativa+frecuencia relativa+dominancia relativa/3) (Cintrón \& Schaeffer-Novelli, 1984). El Índice de Complejidad se calculó por medio de la fórmula: $\mathrm{CI}=$ [densidad total del manglar (árbol $\left.0.1 \mathrm{ha}^{-1}\right)$ x Área Basal Total $\left(\mathrm{m}^{2} 0.1 \mathrm{ha}^{-1}\right)$ $\mathrm{x}$ Altura Promedio del Manglar (m) x Número de Especies] x 10-3 (Pool et al., 1977; Jiménez \& Soto, 1985; Samper-Villarreal et al., 2012).

Análisis granulométrico y salinidad intersticial: En cada parcela se tomó muestras del suelo para realizar análisis granulométrico según la clasificación de Ellison (1997). Para la salinidad del agua intersticial del suelo, se colectó una muestra de agua en un hoyo de $10 \mathrm{~cm}$ de profundidad. Se midió con un refractómetro manual (Model RHS-10ATC).
Análisis Estadístico: Para la comparación de las características estructurales, se realizó un análisis de varianza (ANDEVA) de una vía para las especies presentes en los tres manglares, con pruebas post-hoc de Tuckey en R (Core Team, 2013). Para las especies que sólo se encontraron en dos manglares se compararon utilizando pruebas de $t$ student independientes. Se correlacionó la salinidad del agua intersticial con la altura de los árboles. Para la relación entre el tipo de suelo y las especies encontradas en cada manglar, se realizó la prueba de Hurlbert (Krebs, 1999).

\section{RESULTADOS}

Distribución de las especies de mangle: Se encontraron un total de seis especies de mangle en los sitios estudiados. Las especies de mayor densidad, frecuencia y dominancia, por lo tanto de mayor importancia, fueron consistentes en los tres manglares: $R$. racemosa (mangle blanco) y Pelliciera rhizophorae (mangle piñuela). Las especies Laguncularia racemosa (mangle mariquita), Avicennia germinans (palo de sal), $R$. mangle (mangle rojo) y Conocarpus erectus (mangle botoncillo) mostraron la menor presencia (Cuadro 1).

Estructura de la vegetación: La altura por especie de mangle varió entre los sitios estudiados para $P$. rhizophorae $(\mathrm{F}=91.1 ; \mathrm{gl}=2$; $\mathrm{p}<0.05)$, R. racemosa $(\mathrm{F}=99.4 ; \mathrm{gl}=2 ; \mathrm{p}<0.05)$ y $L$. racemosa $(\mathrm{t}=-2.3 ; \mathrm{p}<0.05)$; con un orden de altura Playa Blanca $>$ Rincón $>$ Escondido. En cambio, la altura de $R$. mangle $(\mathrm{t}=-1.1 ; \mathrm{p}=0.3)$ y A. germinans $(\mathrm{t}=-0.23 ; \mathrm{p}=0.82)$ no mostró variación entre manglares (Fig. 1a).

El DAP de las especies de mangle varió entre los tres sitios para $R$. racemosa $(\mathrm{F}=46.9$; $\mathrm{gl}=2 ; \mathrm{p}<0.05)$, P. rhizophorae $(\mathrm{F}=43.3 ; \mathrm{gl}=2$; $\mathrm{p}<0.05)$, L. racemosa $(\mathrm{t}=-3.0 ; \mathrm{p}<0.05)$ y $R$. mangle $(\mathrm{t}=-7.4 ; \mathrm{p}<0.05)$; en el orden Playa Blanca $>$ Escondido $>$ Rincón. Avicennia germinans es la única especie cuyo DAP no varió entre manglares $(t=-1.7 ; p=0.15)$ (Fig. $1 b)$.

La menor complejidad estructural la presentó el manglar de Rincón (Índice de 
CUADRO 1

Densidad de las especies de mangle, área basal, valor de importancia y total de área muestreada en cada uno de los manglares estudiados. Golfo Dulce, Costa Rica

TABLE 1

Density, basal area, and importance value for each mangrove species and total area sampled in each of the mangroves studied. Golfo dulce, Costa Rica

\begin{tabular}{lccccccccc}
\multicolumn{1}{c}{ Especie } & \multicolumn{3}{c}{ Densidad (árboles ha $\left.\mathrm{hal}^{-1}\right)$} & \multicolumn{3}{c}{ Área basal $\left(\mathrm{m}^{2} \mathrm{ha}^{-1}\right)$} & \multicolumn{3}{c}{ Valor de Importancia $(100 \%)$} \\
& $\mathrm{PB}$ & $\mathrm{R}$ & $\mathrm{E}$ & $\mathrm{PB}$ & $\mathrm{R}$ & $\mathrm{E}$ & $\mathrm{PB}$ & $\mathrm{R}$ & $\mathrm{E}$ \\
Rhizophora racemosa & 307 & 535 & 1117 & 7.13 & 1.46 & 15.43 & 45.0 & 45.0 & 35.0 \\
Pelliciera rhizophorae & 307 & 421 & 2878 & 5.18 & 0.96 & 39.23 & 37.1 & 35.5 & 64.2 \\
Laguncularia racemosa & 82 & 47 & na & 0.56 & 0.15 & na & 7.6 & 5.4 & 0.0 \\
Avicennia germinans & 11 & 42 & na & 0.21 & 0.20 & na & 1.6 & 5.4 & 0.0 \\
Rhizophora mangle & 71 & 125 & na & 0.54 & 0.11 & na & 8.6 & 8.7 & 0.0 \\
Conocarpus erectus & na & na & 9 & na & na & 0.08 & 0.0 & 0.0 & 0.8 \\
Área (ha) & 0.46 & 0.19 & 0.22 & & & & & & \\
\hline
\end{tabular}

$\mathrm{PB}=$ Playa Blanca. $\mathrm{E}=$ Escondido. $\mathrm{R}=$ Rincón. na=no aplica.

A Altura

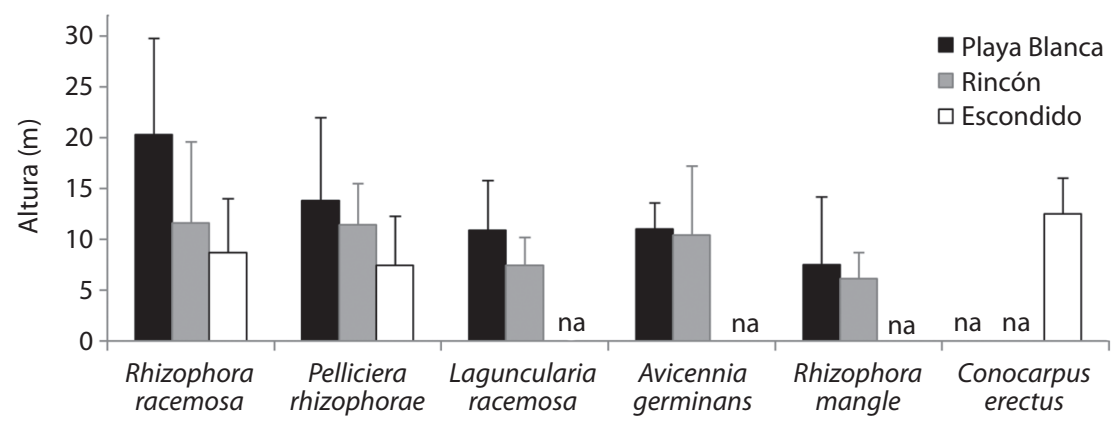

B Diámetro a la Altura del Pecho

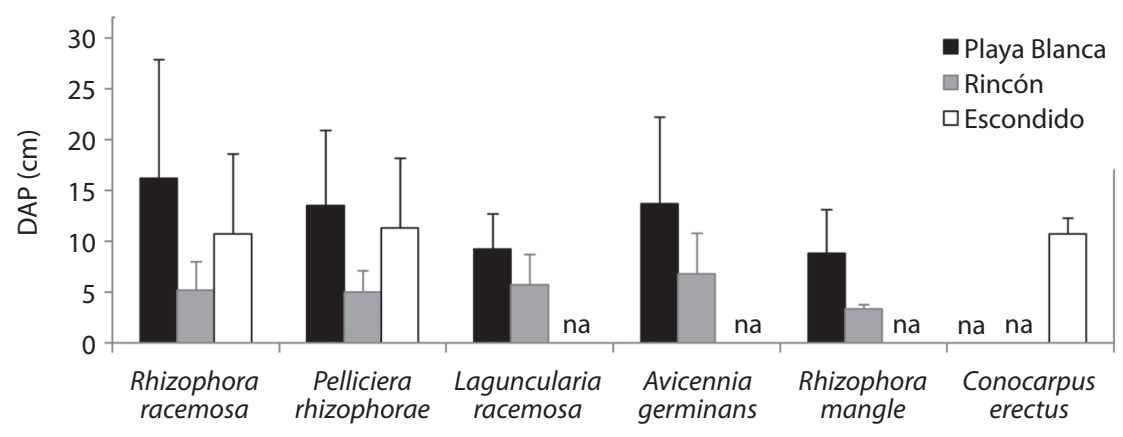

Fig. 1. Altura (A) y diámetro a la altura del pecho (DAP) (B) promedio ( \pm desviación estándar) para cada especie de mangle encontrada en los manglares de Playa Blanca, Rincón de Osa y Escondido, Golfo Dulce, Cosa Rica.

Fig. 1. Mean ( \pm standard deviation) height (A) and diameter at breast height (DBH) (B) average for each mangrove species found in the mangroves of Playa Blanca, Rincón de Osa and Escondido, Golfo Dulce, Cosa Rica. 
Complejidad $[\mathrm{IC}]=2$ ), seguido por Playa Blanca $(\mathrm{IC}=8)$. La mayor complejidad la presentó el manglar de Escondido ( $\mathrm{IC}=51$ ), en el cual si se incluyen las especies de $A$. germinans y R. mangle (observadas fuera de las parcelas) el índice de complejidad asciende a 85 .

a) Playa Blanca: El manglar de Playa Blanca presentó la menor densidad con respecto a los otros manglares estudiados (778 árboles $\mathrm{ha}^{-1}$ ) e incluye un total de cinco especies de mangle: $R$. racemosa, $P$. rhizophorae, $L$. racemosa $R$. mangle y $A$. germinans. Las dos especies más abundantes fueron $R$. racemosa y $P$. rhizophorae, ambas con la mayor densidad (307 árboles ha $^{-1}$ cada especie) (Cuadro 1). Ambas especies se encontraron distribuidas en todo el manglar. $R$. mangle presentó una abundancia regular, predominó en las partes externas de algunos sectores, especialmente cerca de los canales de los ríos. Laguncularia racemosa (medianamente abundante) y A. germinans (la menos abundante) se cuantificaron especialmente en los sectores internos (Fig. 2a).

Un sector de este manglar, cercano al río La Chanchera, presentó tres zonas bien características: la externa que comprendió los primeros 50-60m a partir de la línea de costa, una franja de arena entre 60-80m llamada berma y una zona posterior hasta el interior del manglar. En la parte externa del manglar dominaron parches de $P$. rhizophorae asociada con L. racemosa. En la zona arenosa de la berma, con una salinidad del suelo cero, abundaron especies típicas de vegetación terrestre de las familias Tilliaceae, Fabaceae, Annonaceae, Bignoniaceae y Anacardiaceae. En el sector interno del manglar (después de la berma) y en los sitios protegidos del impacto directo del oleaje abundaron $R$. racemosa, entremezclada con algunos individuos de $A$. germinans y $P$. rhizophorae (Fig. 2a).

Los valores correspondientes a la altura y el diámetro a la altura del pecho (DAP) del dosel (promedio \pm desviación estándar, $15.4 \pm 9.4 \mathrm{~m}$ y $13.4 \pm 9.0 \mathrm{~cm}$, respectivamente) fueron mayores con respecto a los otros dos manglares estudiados. En Playa Blanca, los fustes de $R$. racemosa presentaron las mayores alturas y DAP. Pelliciera rhizophorae y $L$. racemosa mostraron alturas mayores a las de Rincón y Escondido. Avicennia germinans y $R$. mangle presentaron alturas similares a Rincón. Los árboles de $R$. mangle; en particular, constituyen una formación con menor altura y DAP; pero más gruesos que los de Rincón $(R$. mangle no está presente en el área estudiada en Escondido) (Fig. 1). Las áreas basales de $L$. racemosa, A. germinans y $R$. mangle fueron menores en este manglar, aunque las dos primeras mayores que en Rincón. Las especies de mayor área basal fueron $R$. racemosa y $P$. rhizophorae (Cuadro 1).

b) Rincón de Osa: La densidad total del manglar de Rincón correspondió a 1170 árboles $\mathrm{ha}^{-1}$, y se observaron cinco especies de mangle: $R$. racemosa, $P$. rhizophorae, L. racemosa, $R$. mangle y A. germinans. Rhizophora racemosa es la especie dominante (densidad

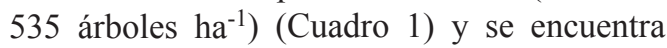
distribuida en las partes externas, medias e internas del manglar (Fig. 2b). Pelliciera rhizophorae también es abundante (Cuadro 1), dispuesta en parches en los sectores medios e internos del manglar, aunque también se encontraron árboles cercanos a la línea de costa. Rhizophora mangle (de abundancia media) se encontró en parches pequeños en la parte media del manglar. Avicennia germinans y L. racemo$s a$ fueron las especies que presentaron menor abundancia y prevalecieron hacia los sectores externos del manglar. En las zonas internas es común encontrar vegetación terrestre asociada a zonas de mangle.

Las especies de mayor área basal en este manglar fueron $R$. racemosa y P. rhizophorae (Cuadro 1). La altura media del dosel $(10.7 \pm 6.4 \mathrm{~m})$ fue menor a la de Playa Blanca pero mayor a la de Escondido. En cambio, el DAP promedio presentó valores menores con respecto a los otros manglares estudiados $(5.0 \pm 2.5 \mathrm{~cm})$. El mayor DAP se midió en árboles de $A$. germinans, seguido por $R$. racemosa, $P$. rhizophorae, $L$. racemosa y $R$. mangle (Fig. 1). 

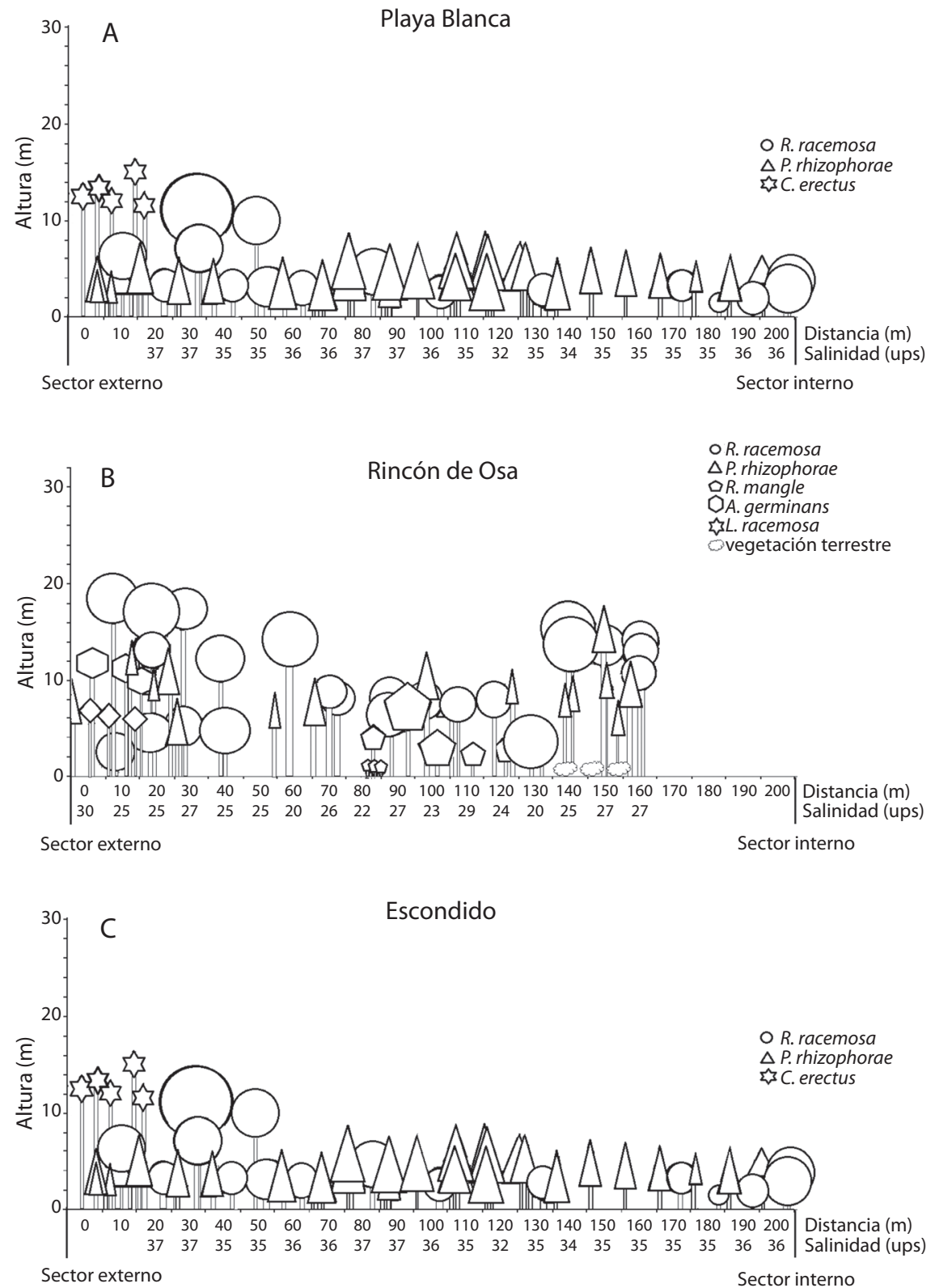

Fig. 2. Perfiles descriptivos de vegetación en los manglares de (A) Playa Blanca; (B) Rincón de Osa; y (C) Escondido, Golfo Dulce, Costa Rica.

Fig. 2. Profiles describing the mangrove vegetation at (A) Playa Blanca; (B) Rincon de Osa; and (C) Escondido, Golfo Dulce, Costa Rica.

c) Escondido: El manglar de Escondido presentó la mayor densidad total de los tres manglares estudiados con 4004 árboles ha-1 (Cuadro 1), notándose la presencia de tres especies de mangle: P. rhizophorae, $R$. racemosa y C. erectus (esta última se encontró únicamente en este manglar). Avicennia germinans y $R$. mangle se observaron en el manglar pero ninguna se cuantificó dentro de las parcelas de estudio. Las dos especies más comunes fueron $P$. rhizophorae y $R$. racemosa, formando parches en diferentes sectores del manglar y con 
las densidades más altas entre los tres manglares estudiados (Cuadro 1). En las partes externas del manglar es común encontrar ambas especies entremezcladas con algunos individuos de C. erectus (poco abundante) (Fig. 2c).

En Escondido se presentaron las áreas basales más altas con respecto a los otros dos manglares, donde $P$. rhizophorae mostró la mayor área basal y Conocarpus erectus la menor (Cuadro 1). En cambio, la altura media del dosel $(7.8 \pm 5.1 \mathrm{~m})$ fue la menor de todos los manglares estudiados; siendo Conocarpus erectus la especie de mayor altura. En este manglar, $R$. racemosa, $P$. rhizophorae y $C$. erectus presentaron DAP similares entre sí, las dos primeras especies con valores intermedios de los tres manglares estudiados $(11.1 \pm 7.2 \mathrm{~cm})$ (Fig. 1).

Tipo de suelo: El sustrato más común en todos los sitios de estudio correspondió al tipo de arena franca y franco. $R$. racemosa se presentó en todos los tipos de suelos, con preferencia por los suelos de arena-franca. Pelliciera rhizophorae presentó la mayor abundancia en el suelo clasificado como arena franca. Avicennia germinans y L. racemosa presentaron preferencia por el suelo franco.

Salinidad: Los manglares estudiados se caracterizan por presentar baja salinidad en el agua intersticial y una homogeneidad en los valores de este parámetro entre la parte externa e interna del manglar (Fig. 2).

\section{DISCUSIÓN}

Este estudio reporta por primera vez la composición florística y estructura de tres manglares en Golfo Dulce, Costa Rica. Los resultados coinciden con lo reportado para los manglares de la región del Pacífico Sur de Costa Rica en presencia y dominancia de especies. Sin embargo, la estructura y complejidad mostró variaciones entre los tres manglares estudiados, más allá de sus similitudes con otros de la región. La distribución de las especies en los manglares estudiados, así como la composición florística, es similar a otros manglares del Pacífico Centroamericano, especialmente en zonas con alta precipitación (Jiménez \& Soto, 1985; Jiménez, 1994a; 1994b; Mainardi, 1996).

Los bosques de mangle del Pacífico Sur de Costa Rica presentan un importante grado de desarrollo, favorecido por el aporte de agua dulce proveniente de la alta precipitación durante casi todo el año (Polanía, 1993). Los manglares del Golfo Dulce típicamente se caracterizan por presentar una mezcla de $R$. mangle, $R$. racemosa y $P$. rhizophorae (Silva, 2009), observaciones que concuerdan con los resultados del presente estudio.

La dominancia de $P$. rhizophorae en los manglares de Playa Blanca, Escondido y Rincón de Osa, coincide con la distribución geográfica de esta especie, la cual está restringida a la costa Pacífica de América, con su máxima representación en los manglares del Pacífico Sur de Costa Rica (Jiménez, 1994a; 1994b). En el manglar de Escondido la composición de especies es diferente a los otros dos manglares, ya que es el único manglar en que se encontró $C$. erectus y en el cual no se encontraron tres especies que sí estaban presentes en los otros manglares (A. germinans, L. racemosa y $R$. mangle). Laguncularia racemosa y $C$. erectus, fueron componentes minoritarios en los manglares estudiados, lo cual coincide con lo anteriormente mencionado para los bosques de mangle de la región (Jiménez 1994a; 1994b).

En los manglares estudiados no se identificó una zonación definida con respecto a la presencia de especies. Esta falta de zonación es contraria a la reportada para los manglares del Pacífico Norte (Jiménez, 1994a; 1994b) y podría deberse a la alta precipitación en el Pacífico Sur (Jiménez, \& Soto, 1985; IMN, 2013). Los tres manglares estudiados parecieran ser semejantes en: i) la dominancia de dos especies ( $R$. racemosa y $P$. rhizophorae), ii) características estructurales (altura y DAP para $A$. germinans, y altura para $R$. mangle), y iii) falta de zonación marcada de especies. Sin embargo, la altura y el diámetro a la altura del pecho (DAP) de varias de las especies de mangle sí 
presentaron variaciones entre los manglares y la estructura general y composición de especies de cada bosque pareciera ser diferente, en particular la de Escondido.

Las diferencias en el desarrollo estructural, pueden reflejar variaciones en la amplitud de la marea, gradientes de salinidad, textura y estabilidad del suelo, condiciones climáticas y aporte de nutrimentos (Delgado, Hensel, Jiménez, \& Day, 2001; Lovelock, Feller, McKee, \& Thompson, 2005). La presencia y grado de desarrollo de cada especie de mangle depende por lo general de su capacidad para soportar diferentes grados de salinidad (Hogarth, 1999). Sin embargo, en los tres manglares la salinidad del suelo no parece constituir un factor determinante en la distribución de las especies, ni en la complejidad estructural de los manglares estudiados, ya que se desarrollan en condiciones climáticas y de inundación mareal similares. En general, se considera que estos manglares no están limitados por deficit de agua dulce y altas salinidades, contrario a los manglares del Pacífico Norte (Jiménez 1994a; 1994b). El tipo de suelo también fue similar entre los manglares y no se considera un factor que ayude a explicar las variaciones entre manglares. Debido a que la salinidad intersticial y el tipo de suelo entre los manglares son similares y los tres sitios están expuestos a una prolongada estación lluviosa; la variación en los atributos estructurales puede deberse a otros factores no considerados en este estudio o a impactos antropogénicos, como incrementos en la entrada de nutrientes o sedimentos por las cuencas asociadas a estos bosques.

Bosques con alturas y diámetros mayores, y con menores densidades de árboles están asociados a bosques maduros, con un buen desarrollo estructural (Cintrón \& Schaefer-Novelli, 1983; Jiménez, Lugo, \& Cintrón, 1985; Fromard et al., 1998). Los manglares de Rincón y Playa Blanca presentan índices que reflejan menor complejidad ( $\mathrm{IC}=2$ y 8 , respectivamente). Sin embargo, el manglar de Playa Blanca es el manglar que mejor se asocia a un manglar maduro, pues los árboles presentaron mayores alturas y diámetros y menor densidad con respecto a los otros manglares estudiados. Los bajos índices de Rincón y Playa Blanca reflejan una menor densidad y reducida área basal de los árboles presentes en estos sitios. Los índices de estos dos manglares son menores a otros reportados para el Pacífico (Jiménez \& Soto, 1985; Samper-Villarreal et al., 2012); aunque mayores con respecto al manglar de Panamá en el Pacífico Norte $(\mathrm{IC}=1,1)$ (Samper-Villarreal et al., 2012). Por el contrario, el manglar de Escondido presentó el mayor índice de complejidad de los tres sitios estudiados ( $\mathrm{IC}=51$ 85) y uno de los más altos reportados para el Pacífico de Costa Rica, similar a los manglares de Quepos en el Pacífico Central $(\mathrm{IC}=65.3)$ y Sierpe en el Pacífico Sur (IC=54.3) (Jiménez \& Soto, 1985) y al manglar de Iguanita en Bahía Culebra ( $\mathrm{IC}=86.5)$ (Samper-Villarreal et al., 2012). Sin duda, parte de la alta complejidad de Escondido se debe a la mayor densidad estimada para dichos estudios de índices de complejidad en el país. El manglar de Escondido, presentó el mayor número de individuos y las áreas basales más altas, especialmente para P. rhizophorae; sin embargo, presentó alturas y diámetros menores que Rincón y Playa Blanca; lo cual es característico de manglares de formación más reciente. Si se comparan rodales de $R$. racemosa y $P$. rizophorae con otros rodales de estas mismas especies en el Pacífico Sur de Costa Rica, como el manglar de Térraba-Sierpe (Lahmann, 1993; Jiménez, 1994a; 1994b; Mainardi, 1996), es evidente el reducido desarrollo estructural de ambas especies en los manglares del Golfo Dulce.

El índice de complejidad es indicativo de la complejidad estructural de un bosque; sin embargo, debe ser analizado considerando las variables que lo componen (número de especies, altura promedio, área basal y densidad) (Jiménez et al., 1985; Fromard et al., 1998). El análisis histórico o paleoecológico de la formación y desarrollo de cada uno de los manglares estudiados, así como la estabilidad en sus condiciones hidrológicas actuales, aportaría información importante para discernir variaciones en la madurez de cada manglar que puedan 
ayudar a explicar su estructura y composición florística actual.

Los manglares estudiados, son similares en la dominancia de $R$. racemosa y $P$. rhizophorae. Sin embargo, presentan diferencias estructurales lo que podría sugerir que otros factores diferentes a la salinidad, tipo de suelo, escorrentía y precipitación, pueden estar determinando las variaciones en el desarrollo estructural que presentan entre sí. Estudios geomorfológicos y topográficos, entre otros, podrían aclarar las causas de variaciones en estructura y complejidad encontrada actualmente entre los manglares de Playa Blanca, Escondido y Rincón de Osa.

\section{AGRADECIMIENTOS}

Las conclusiones presentadas en este artículo representan los resultados de los proyectos números 605-A4-954 y 808-A2-129 de la Universidad de Costa Rica, adscritos a la Escuela de Biología y al Centro de Investigación en Ciencias del Mar y Limnología (CIMAR). Se agradece el apoyo a los miembros de la Asociación Protectores del Manglar de Playa Blanca de Puerto Jiménez (ASOMANGLE) por la colaboración durante el trabajo de campo. Jeffrey Sibaja realizó los análisis estadísticos del suelo. Se agradece la colaboración de los estudiantes que trabajaron para el proyecto como asistentes David Mena, Alexander Casti1lo, Miguel Artavia y Andrey Villalobos.

\section{RESUMEN}

Este estudio reporta las características estructurales y distribución de las especies en los bosques de mangle de Playa Blanca, Escondido y Rincón en Golfo Dulce, Pacífico Sur de Costa Rica. Se realizaron múltiples transectos en cada uno de los manglares. Cada $10 \mathrm{~m}$ a lo largo de cada transecto se delimitaron parcelas cuadradas de $5 \times 5 \mathrm{~m}$. En cada parcela se midió el diámetro a la altura del pecho y altura de los árboles de mangle y se tomaron muestras de sedimento y de agua intersticial (salinidad). Se determinó la densidad total y por especie para cada manglar, el área basal y valor de importancia por especie y la complejidad general entre los manglares. Las especies dominantes en los tres manglares estudiados fueron Rhizophora racemosa y Pelliciera rhizophorae. Los manglares de Playa Blanca y Rincón presentaron mayor similitud en la estructura del bosque entre sí que con el manglar de Escondido. Playa Blanca fue el bosque de mayor altura del dosel y menor densidad; mientras que Escondido presentó mayor densidad y menor altura, así como el menor número de especies y el único donde se encontró la especie Conocarpus erectus. La composición de especies presente en estos manglares coincide con la reportada para la región. Sin embargo, la variación en estructura y complejidad entre los tres manglares es mayor a las similitudes con otros de la región. El estudio histórico y topográfico de estos manglares puede aclarar las variaciones en estructura y complejidad encontrada actualmente entre ellos.

Palabras clave: manglar, Rhizophora, Pelliciera, Avicennia, Laguncularia, Conocarpus, Península de Osa.

\section{REFERENCIAS}

Caribbean Coastal Marine Productivity (CARICOMP). (2001). Manual of methods for mapping and monitoring of physical and biological parameters in the coastal zone of the Caribbean. Kingston, Jamaica: CARICOMP Data Management Center, University of the West Indies. Retrieved from http://www.ima.gov. tt/home/ images/stories/caricomp_manual_2001.pdf

Cintrón, G. \& Schaefer-Novelli, Y. (1983). Introducción a la ecología del manglar. Montevideo, Uruguay: Oficina regional de Ciencia y Tecnología UNESCO para América Latina y el Caribe ROSTLAC.

Cintrón, G. \& Schaeffer-Novelli, Y. (1984). Methods for studying mangrove structure. In S. C. Snedaker \& J. G. Snedaker (Eds.), The mangrove ecosystem: research methods (pp. 91-113). United Kingdom: The Chauser Press.

Cintrón-Moreno, G., \& Schaeffer-Novelli, Y. (1992). Ecology and management of New World mangroves. In U. Seeliger (Ed.), Coastal plant communities of Latin America (pp. 233-258). San Diego, USA: Academic Press.

Córdoba-Muñoz, R., Romero-Araya, J. C., \& WindevoxhelLora, N. J. (1998). Inventario de los humedales de Costa Rica. San José, Costa Rica: UICN, MINAE, SINAC, Embajada Real de los Países Bajos.

Delgado, P., Hensel, P., Jiménez, J. A., \& Day, J. D. (2001). The importance of propagule establishment and physical factors in mangrove distributional patterns in a Costa Rican estuary. Aquatic Botany, 71, 157-178.

Donato D. C., Kauffman, J. B., Murdiyarso, D., Kurnianto, S., Stidham, M., \& Kanninen, M. (2011). Mangroves among the most carbon-rich forests in the tropics. Nature Geoscience, 4, 293-297.

Echeverría-Sáenz, S. (2009). Uso de la tierra y transporte de sólidos en suspensión en la cuenca del rio Rincón, Península de Osa, Puntarenas, Costa Rica 
(Tesis de Maestría). Universidad Estatal a Distancia, Costa Rica.

Ellison, J. (1997). Mangrove community characteristics and litter production in Bermuda. In B. Kjerve, L. D. Lacerda, \& E. S. Diop (Eds.), Mangrove ecosystem studies in Latin America and Africa (pp. 8-17). Paris, France: UNESCO.

Fromard, F., Puig, H., Mougin, E., Marty, G., Betoulle J. L., \& Cadamuro, L. (1998) Structure, above-Ground Biomass and Dynamics of Mangrove Ecosystems: New Data from French Guiana. Oecología, 115, 39-53.

Hogarth, P. (1999). The biology of mangroves. New York, USA: Oxford University Press.

Instituto Meteorológico Nacional de Costa Rica (IMN). (2013). Atlas Climatológico. Disponible en http:// www.imn.ac.cr

Jiménez, J. A., Lugo, A. E., \& Cintrón, G. (1985). Tree Mortality in Mangrove Forests. Biotropica, 17, 177-185.

Jiménez, J. A. \& Soto, R. (1985). Patrones regionales en la estructura y composición florística de los manglares de la Costa Pacífica de Costa Rica. Revista de Biología Tropical, 33, 25-37.

Jiménez, J. A. (1994a). Los manglares del Pacífico Centroamericano. Heredia, Costa Rica: EFUNA.

Jiménez, J. A. (1994b). Bosques de manglares en la costa Pacífica de América Central. Revista Forestal Centroamericana, 3, 13-17.

Jiménez, J. A. (1999). Ambiente, distribución y características estructurales en los manglares del Pacífico de Centro América: contrastes climáticos. In Y. Arancibia, \& A. L. Lara-Domínguez (Eds.), Ecosistemas de manglar en América Tropical (pp. 51-70). Veracruz, México: Instituto de Ecología, A.C.

Kathiresan, K. \& Qasim, S. Z. (2005). Biodiversity of mangrove ecosystems. New Delhi, India: Hindustan Pub. Corp.

Krebs, C. J. (1999). Ecological Methodology (2 $2^{\text {nd }}$ ed.). California, USA: Addison-Welsey.

Lahmann, E. (1993). The mangrove forests of Sierpe, Costa Rica,. In T. J. Davis (Ed.), Towards the Wise Use of Wetlands (pp. 82-88). Gland, Switzerland: Ramsar Convention Bureau, Wise Use Project.

Lovelock, C. E., Feller, I., McKee, K. L., \& Thompson, R. (2005). Variation in mangrove forest structure and sediment characteristics in Bocas del Toro, Panamá. Caribbean Journal of Science, 41, 456-464.

Mainardi, V. (1996). El manglar de Térraba-Sierpe en Costa Rica. Serie Técnica, Informe Técnico 284. Turrialba, Costa Rica: Centro Agronómico Tropical de Investigación y Enseñanza (CATIE).

Ministerio de Ambiente, Energía y Telecomunicaciones (MINAET). (2013). Mapa de tipos de bosque de Costa Rica. San José, Costa Rica: Inventario Nacional Forestal.
Nielsen-Muñoz, V., \& Quesada-Alpízar, M. A. (2006). Informe Técnico Ambientes Marino Costeros de Costa Rica. San José, Costa Rica: Comisión Interdisciplinaria Marino Costera de la Zona Económica Exclusiva de Costa Rica, CIMAR, CI, TNC.

Pizarro, F., \& Angulo, H. (1994). Diagnóstico de los manglares de la costa Pacífica de Costa Rica. In O. D. Suman (Ed.), El ecosistema de manglar en América Latina y la Cuenca del Caribe: su manejo y conservación (pp. 33-63). Miami, USA: Universidad de Miami.

Pizarro, F., Piedra, L., Bravo, J., Asch, J., \& Asch, C. (2004). Manual de procedimientos para el manejo de los manglares. Heredia, Costa Rica: EFUNA.

Polanía, J. (1993). Mangroves of Costa Rica. In Conservation and sustainable utilization of mangrove forests in Latin America and Africa regions (pp. 129-137). Okinawa, Japan: ITTO/ISME.

Pool, D. J., Snedaker, S. C., \& Lugo, A. E. (1977). Structure of mangrove forests in Florida, Puerto Rico, México and Costa Rica. Biotropica, 9, 195-212.

Quesada-Alpízar, M. A., \& Cortés, J. (2006). Los ecosistemas marinos del Pacífico sur de Costa Rica: Estado del conocimiento y perspectivas de manejo. Revista de Biología Tropical, 54 (Supp. 1) 101-145.

R Development Core Team (2013). A language and Environment for Statistical Computing. Versión 3.0.1. Vienna, Austria: R Foundation for Statistical Computing.

Samper-Villarreal J., Cortes, J., \& Benavides-Varela, C. (2012). Description of the Panamá and Iguanita mangrove stands of Bahía Culebra, North Pacific coast of Costa Rica. Revista de Biología Tropical, 60 (Supp. 2) $109-120$

Satyanarayana, B., Raman, A. V., Dehairs, F., Kalavati, C., \& Chandramohan, P. (2002). Mangrove floristic and zonation patterns of Coringa, Kakinada Bay, East coast of India. Wetlands Ecology and Management, 10, 25-39.

Silva, M. (2005). Manglares de Golfito. In J. Lobo \& F. Bolaños (Eds.), Historia natural de Golfito (pp. 55-65). San José, Costa Rica: INBio.

Silva, M. (2009). Mangroves of Costa Rica,. In J. Cortés \& I.S. Wehrtman (Eds.), Marine Biodiversity of Costa Rica, Central America (pp. 123-130). Berlin, Germany: Springer.

Soto, R., \& Jiménez, J. A. (1982). Análisis fisionómico y estructural del manglar de Puerto Soley, La Cruz, Guanacaste, Costa Rica. Revista de Biología Tropical, 30, 161-168.

Spalding, M., Spalding, M., Kainuma, M., \& Collins, L. (2010). World atlas of mangroves. Earthscan, Malta: Gutemberg Press.

Tomlinson, P. B. (1994). The botany of mangroves. New York, USA: Cambridge University Press.

Zamora-Trejos, P. \& Cortés, J. (2009). Los manglares de Costa Rica: el Pacífico Norte. Revista de Biología Tropical, 57, 473-488. 